\title{
Modular Approach for Face Recognition System using Multilevel Haar Wavelet Transform, Improved PCA and Enhanced Back Propagation Neural Network
}

\author{
Prachi Agarwal \\ Assistant Professor, Department of CS \& IT \\ Moradabad Institute of Technology \\ Moradabad
}

\author{
Naveen Prakash \\ Assistant Professor, Department of CSE \\ I.F.T.M. University \\ Moradabad
}

\begin{abstract}
With rapidly developing technology, it is important to invent an efficient and effective security system that will help to detect unauthorized access on any system. Hence it is essential necessary to implement a highly secure, economic and reliable face recognition system to enable the protection of computer systems from unauthorized access. So, in this paper a fast efficient approach is proposed for the recognition of human faces. Thus, firstly Haar wavelet transform is implemented for multilevel decomposition of face image into several subband images. The decomposed image subbands are then used as input by Improved Principal Component Analysis (IPCA) approach for extracting features with the help of eigenvalues and Eigen vectors. Then classification of features using Back Propagation Neural Network (BPNN) is done and finally image is being recognized comparing it to the testing images existing in the images database. More efficient BPNN is used to improve the recognition rate and to overcome the problems associated with variations in illumination and poses. Thus feed forward neural network shows the effectiveness of the proposed algorithm.
\end{abstract}

\section{General Terms}

Recognition, Security, Algorithms

\section{Keywords}

Eigen vectors, Improved Principal component analysis (IPCA), Feature extraction, Discrete Wavelet Transform.

\section{INTRODUCTION}

In the current scenario, biometric authentication technologies are increasingly used in practical applications. In comparison to other types of recognition such as fingerprint analysis and iris scanning, facial recognition is a more favorable approach and is non- interfering. The face recognition system extends more natural and more prosperous approach for the identification and authentication of human beings in comparison to the preexisting biometric security technologies. Hence, face recognition is the most crucial part in biometrics methods that are being used for the identification of individuals extracting the facial features.

Face recognition system [1] is a computer visual sensation which automatically discovers a human face from existent database images. The face recognition emersion is aspiration because it requires looking after all potential appearing variances induced by modifications in illumination, facial features and expressions, viewing directions or poses, aging and conceals like spectacles or cosmetics.

The designing of prominently executing algorithms for automatic facial recognition systems for implementing on real life applications is very challenging. There are various flourishing applications of face recognition, such as biometric personal recognition, human-to-machine interaction and access control. Some areas of commercial concern include law enforcement and police investigation, video investigation for security, airport security, and recognition at front entrance for home safety, security and acknowledgment at ATM or in alignment with a smart card for authentication.

Basically, the facial recognition system can be depicted with the help of the following block diagram.

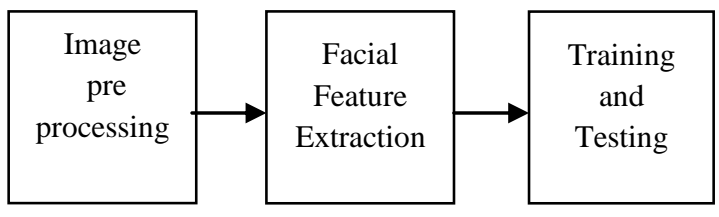

Fig 1: Steps of face recognition system

1) Image Pre-processing: The first phase involves face detection, normalization and removal of background, noise and other components of the face that can affect the recognition rate. The image is converted into a gray scale image and is resized to a pre-determined standard. Discrete Wavelet Transform (DWT) can be used to perform face expression normalization.

2) Facial Feature Extraction: In this phase, the face image being normalized serves as input to the feature extraction module to find the fundamental features to be used for 
further classification. The module contains a feature vector that can be used to represent the face image.

3) Training and Testing: At this stage, Neural Network, Euclidean Distance (ED), Hamming Distance or Support Vector Machine (SVM) techniques can be employed to train the system followed by testing the images for recognition.

There are many techniques formulated for face recognition. These recognition algorithms can be described into following categories: local approaches, holistic or global feature approaches and hybrid approaches. The local approaches of face recognition are used for the segmentation of local facial features such as eyes, lips, nose, eyebrows, chin and the spatial relationships among them. Afterwards these facial features are feed as input data for morphological classification. The approach needs a vast collection of database images for efficient training hence enhancing the computation time. Whereas when holistic approach is used, entire face region is saved as input data in face detection system. But it is sensitive to translation, rotation and poses changes. Since, local and global approaches do not provide a complete representation of the facial image, a hybrid features based face recognition system may be projected that combines the two former approaches into a single system.

In many face recognition systems, the artificial neural networks are widely used as the classifier. While figuring out the problems associated with the use of multilayered feed forward neural networks, first determine the number of hidden neurons layer.

\subsection{Discrete Wavelet Transform}

The Discrete Wavelet Transform (DWT) [2] is the most familiar decomposition approach which is used for image processing. It breaks down an image into a set of certain functions called wavelets and this decomposition is known as the resolution of the image. The two-dimensional DWT is applied as a set of filter banks that consists of a cascaded strategy of low pass filters and high pass filters. The received output is the decomposition of the input image into four multi-resolution sub-bands: LL, LH, HL and $\mathrm{HH}$. The LL subband is an estimation of the original image. The subband LH represents the alterations in the image along horizontal directions whereas the subband HL represents alterations along vertical directions. The subband $\mathrm{HH}$ shows the higher frequency factor of the image. This first level decomposition can be further followed through the LL subband for more decomposition levels.

\subsection{Facial Feature Extraction Process}

Facial features extraction localizes the most peculiar face elements (mouth, eyes, nose, etc.) within the human face images. It initializes various face processing techniques like face recognition, face tracking or the facial expression recognition. Improved Principal Component Analysis (IPCA) is used as the facial feature extractor. IPCA is implemented on the outcome received after the process of face image decomposition to obtain eigenvector. The original image can be recovered using prevailing eigenvector. So it is a very good approach to represent the original image in the form of eigenvector. This eigenvector is selected from the highest eigenvalues that contains variances in the original dataset.
The earlier approach of PCA [3] computes the primary component which is the position of highest covariance value among the feature vectors. But now IPCA approach is used in which one thing to be considered is that if the feature vectors have small differences in their elementary values than those features are the facial features between various faces. Whereas, if the feature vectors have larger differences in their elementary values that such features are not part of the facial features e.g. noise. Thus the elements having smaller feature vector covariance are facial features whereas the elements having larger values of covariance between the feature vectors are not the facial features. In the former PCA approach many learning patterns are needed, because the method reserved some other elements that are not feature. Hence, this improved approach is proposed to select the elements having smaller difference in feature vectors and consider it to be the primary factor of the algorithm.

\subsection{Face Recognition using Back-Propagation Neural Network}

During the training phase, supervised training process is used to adjust Neural Network weights and this procedure is known as back propagation (BP). Back propagation [4] is a gradient declination technique used to find a satisfactory local minimal in the NN weight space to obtain the minimal error.

Back Propagation Neural Network (BPNN) is a feed forward and multilayered Neural Network. It contains input layer followed by one or more hidden layers which are further being complied by the final output layer.

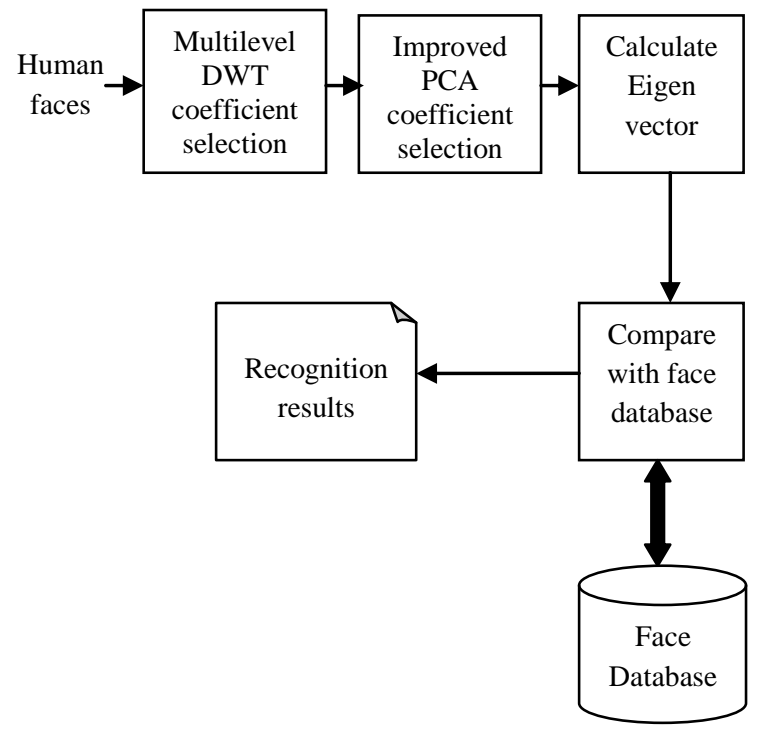

Fig 2: Selection of coefficients and recognition of features

\section{LITERATURE REVIEW}

The security of the existing systems needs to be designed efficiently for controlling the unauthorized accesses. So, designing an efficient face recognition system is very important. Mayank Agarwal et al. [5] presented an approach for face recognition based on scientific theory approach of encrypting and decrypting the facial image. The suggested method had two stages- First stage includes 
principle component analysis for the extraction of the facial features. The next stage involved the use of the feed forward back propagation Neural Network for face recognition. The paper presented face recognition system using combination of PCA approach and BPNN technique. They also showed comparison between the efficiency of the system to K-means, Fuzzy Ant with fuzzy C-means resulting in better recognition rate compared to the other two techniques.

Raman Bhati et al. [6] represented the feature vectors with the help of Eigenfaces. They proposed a technique for the selection of the learning rate in a single layer feed forward neural network and BPNN also. The results of the proposed approach were good in all aspects including mean square error, identification rate and learning time. According to the paper if correct learning rate is assigned to a network the single layer neural network can yield $100 \%$ recognition accuracy. Wavelets were used for feature extraction.

Jong-Min Kim et al. [7] proposed a face recognition method to improve the recognition rate by combining the multi-layer neural network with the approach that calculates the minor count of Eigenface feature vector using PCA approach. They suggested a method with improved functionality having variances in illumination and noise in order to minimize the recognition error. They applied the rectangular feature technique to extract the face from the background image. Then face recognition was performed by reducing the size of the image and after the removal of the noise from the image. Thereafter the proper value of vector was calculated with the help of PCA and finally the resultant image was passed on to the multi-layer neural network. Thus the performance of conventional PCA recognition method was compared to the approach using combined features of PCA and MLNN.

Amir Benzaoui et al. [8] suggested a recognition system of face detection which includes two stages: learning and detection. In the first stage, all face and non-face pictures were changed into gray level pictures and then these gray level pictures were filtered by median filter in order to reduce noise. Then feature were extracted by selecting appropriate information from every single image so that data can be collected for classification learning. After that they used Discrete Cosine Transform (DCT) and extracted a vector value of appropriate coefficient which represented the essential parameters for simulating the original image.

Ki-Chung Chung et al. [9] suggested a new face recognition method based on Gabor filtering responses and used Principal Component Analysis (PCA). In this approach Gabor filtering was employed on predefined fiducial points to match robust facial features out of the original face image. After that transformation of the facial features was done into eigenspaces by PCA so that optimal classification could be achieved for human facial representations.

M. Koteswara Rao et al. [10] proposed a face recognition system using Discrete Wavelet Transform (DWT) \& eigenvectors. The image was decomposed into subbands and then PCA was used for processing the subbands. PCAh approach assisted in reducing high dimensionality to low dimensionality. The sub images were rearranged into vertically and horizontally matrices. The global feature vectors were yielded and then utilized for face recognition. The horizontal and vertical variances of the feature vector were also viewed. The proposed method contributed to improved intermediate recognition rate and less computation time in comparison to the other existing methods.

Swarup Kumar Dandpat et al. [11] proposed a system designed for high performance of automatic facial recognition system. They targeted to develop an efficient computational approach for precise face recognition. A high performance automatic facial recognition system was designed with the help of conventional Principal Component Analysis (PCA). The efficiency of the system improved a lot and better recognition performance was achieved due to less computational cost incurred compared to other existing systems.

Himanshu S. Bhatt [12] proposed a face recognition system using Eigen faces and the system was accurate and fast. When both back propagation neural network was combined with PCA technique in the proposed system, non linear face images were very easily recognized. Hence result was that the method had only few seconds execution time and assured more than $90 \%$ acceptance ratio.

\section{PROBLEM DEFINITION}

Computer systems act as a crucial part in today's life and in the society. Humans are becoming more and more influenced with the enhancing technology as the leaders of this fast evolving technology. Main aim of computer vision is to achieve the power of visual recognition same as that of human beings. Face recognition has achieved much priority in current scenario in the computer vision system. Face recognition demands the evolution of executable technologies to support the development of robust face recognition system. So, the need of the time is to design a robust system that can protect our surrounding 100\% securely from the intruders and all the problems related to recognition of human and the verification of face images can be solved.

\section{PROPOSED METHODOLOGY}

The proposed methodology complies three phases for the robust and efficient recognition of the face images [13]. First phase is the Haar wavelet transform. In this the input image is decomposed into several sub band images. Secondly, feature extraction is done using PCA approach by calculating eigenface values. After that classification of the images is done using the back propagation neural network. Also, the effect of number of hidden layers is established.

\subsection{Multilevel Haar Wavelet Transform}

Haar wavelet transform decomposes the original greyscale image into four regions. These are the higher horizontal frequencies, higher vertical frequencies, higher diagonal frequencies and the regional approximation frequencies. Every region contains certain frequency features but the minimum are contained in the approximation region. This is the first level of image decomposition. The upper left corner approximation region of image is further decomposed into four smaller regions at the next level of decomposition and this process continues. As this is orthogonal transform so the energy of the image is matched up with the energy of the given coefficients. In the below given figure the values $\mathrm{VF}_{5}, \mathrm{VF}_{6}, \mathrm{VF}_{7}$ represents vertical 
components while the horizontal components are represented by $\mathrm{HF}_{1}, \mathrm{HF}_{2}, \mathrm{HF}_{3}$ and $\mathrm{HF}_{4}$.

\begin{tabular}{|c|c|c|}
\hline $\mathrm{HF}_{1}$ & $\mathrm{HF}_{3}$ & \multirow{2}{*}{$\mathrm{VF}_{5}$} \\
\cline { 1 - 2 } $\mathrm{HF}_{2}$ & $\mathrm{HF}_{4}$ & \\
\hline & \\
$\mathrm{VF}_{6}$ & $\mathrm{VF}_{7}$ \\
& \\
\hline
\end{tabular}

Fig 3: Haar wavelet transform showing decomposition of sub-image bands

Algorithm for Haar Wavelet Transform -

1. First of all select an input image of size $\mathrm{n} \times \mathrm{n}$.

2. Set the value of $d$, where $d$ is the number of decomposition levels. Calculate the values of the Approximate coefficient storage, the Horizontal coefficient storage. Also the Vertical coefficient storage along with the Diagonal coefficient storage is calculated.

3. Apply vertical transformation i.e. apply 1-D Haar transform corresponding to each column of the image matrix.

4. After that apply 1-D horizontal transform for finding the resultant scale matrices.

5. Repeat this process for all sub-parts of the image until n-level of decomposition is reached and apply Haar wavelet transform.

6. At the next step resize the given input image matrix to a determined range for display.

7. Then display all the wavelet coefficients in the form of a complete level decomposed image.

8. Now compute the signature matrix of the whole database and then convert it into an unsigned 8bit integer.

9. Now these image subband are used for further processing.

\subsection{Improved Principal component analysis}

Principal component analysis (PCA) transforms the correlated variables in uncorrelated variables called principal components. PCA is a statistical holistic based approach used to determine the principal components of a defined set of images and correspond to each face image's higher dimensions in the form of lower dimensional face space points using few eigenvectors which in turn results in high eigenvalues. In PCA the total number of images stored in the training database is more significant in comparison to the size of the image. Therefore, on increasing the count and form of images in the covariance matrix recognition rate can be enhanced. Face recognition using PCA depends on the information theory approach which extracts relevant information about a face image as efficiently as possible. Hence, it cuts down the dimensionality by proposing the points onto the principal axes and the direction of best level covariance aligns with the set of orthonormal points. The basic face database contains same size and configuration training patterns.

\section{Algorithm for Principal component analysis -}

1. Firstly all the training $2 \mathrm{D}$ images of the database are coordinated and converted into $1 \mathrm{D}$ vector and numbers are allocated to every image of database.

2. Now convert $1 \mathrm{D}$ vector of the images into $2 \mathrm{D}$ matrix.

3. After that select the most distinguishable features among the face images of training database.

4. Next calculate the average value of face images $x^{\prime}=(1 / n) * \operatorname{sum}\left(x_{i}\right)$

where $x=\left\{x_{1}, x_{2}, x_{3}, \ldots, x_{n}\right\}^{T}$ is the feature vector, $\mathrm{n}$ is the number of values and $\mathrm{x}^{\prime}$ is the average value.

5. Now scale the image by reducing the noise influence from that image as $\mathbf{u}=\mathbf{x} / \mathbf{x}$.

6. Now deviation of each image is calculated from its mean image by measuring the variance of mean value with every image existing in training data set and then concatenates all the centered images.

7. Calculate the eigenvectors of the minimized covariance matrix as $\Delta=\mathrm{A}^{\mathrm{T}} \sum \mathrm{A}$

where $\mathrm{A}$ is the matrix for transformation and $\sum$ is the matrix for covariance of the learning patterns.

8. Now the above resulting faces are compared by projecting the centered images into face space.

\subsection{Enhanced Back Propagation Neural Network}

Neural Networks (NNs) are non-analogous mathematical function structures that are based on the working of the human brain. These networks are computational building blocks propelled by discovered procedure in natural networks of biological neurons in the human brain. They comprise simple calculating units known as neurons that are enormously coordinated.

BPNN is multilayered feed forward Neural Network. The BPNN learning algorithm averages the weight values and the values of bias of all neurons during training process. It is done in order to minimize the Mean Square Error (MSE) amongst the targets and thus correctly predicts the desired output.

\section{Algorithm for BPNN -}

1. Firstly set all the values of weights to a random value between -1.0 to 1.0 and the input binary values pattern are set into the neurons of input layer.

2. To activate neurons at all layers multiply the values of weights to the preceding neurons outputs.

3. Then compute the output value of the neuron by moving over the resultant to activation function and repeat it until the final output layer is reached.

4. Now the calculated output pattern is comparable to the desired objected pattern and mean square error is calculated.

5. After that weights are changed using the formula: 
Weight (old) + Learning Rate * Output Error * Output (Neuron i) * Output (Neuron i + 1) * (1 - Output (Neuron i + 1))

where Weight(old) denotes the weight of the previous layer, Learning Rate is the rate required to train the database, Output Error represents the previous steps error.

6. And finally match all the output patterns to the desired patterns achieved in the execution of the algorithm.

\section{STRUCTURAL SUMMARIZATION OF THE PROPOSED ALGORITHM USING FLOW CHART DIAGRAM}

\subsection{ER Diagram to input data in face recognition system}

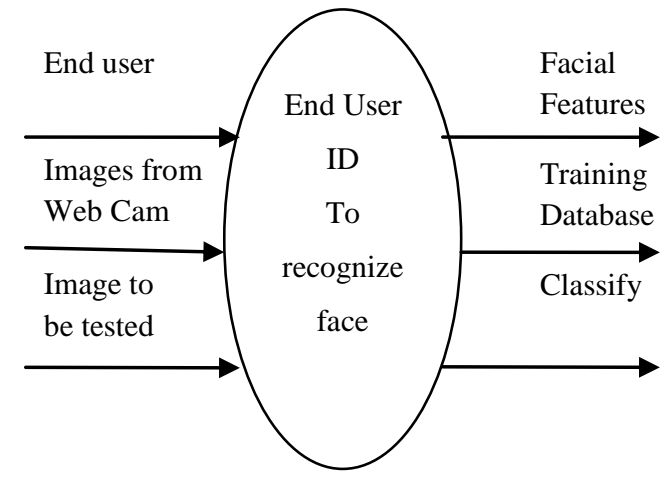

Fig 4: Data Flow Diagram for input

\subsection{Representation for training phase}

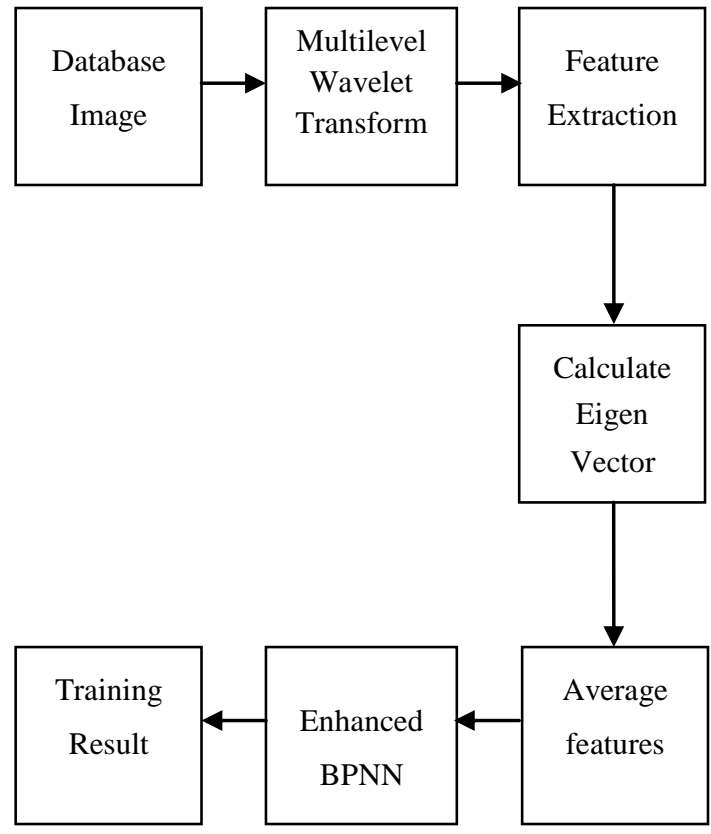

Fig 5: Training phase block diagram

\subsection{Representation for testing phase}

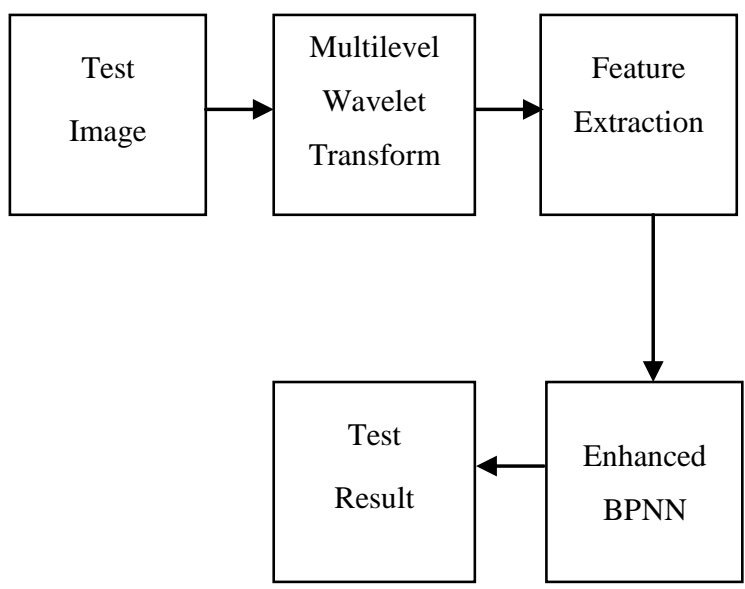

Fig 6: Testing phase block diagram

\subsection{Flowchart for proposed Face Recognition System}

The following flowchart shows the different phases of proposed face recognition system and shows how input image is passed through various phases. Then finally the selected inputted face image is matched with the test image databases and resultant image after recognition is displayed as the final output.

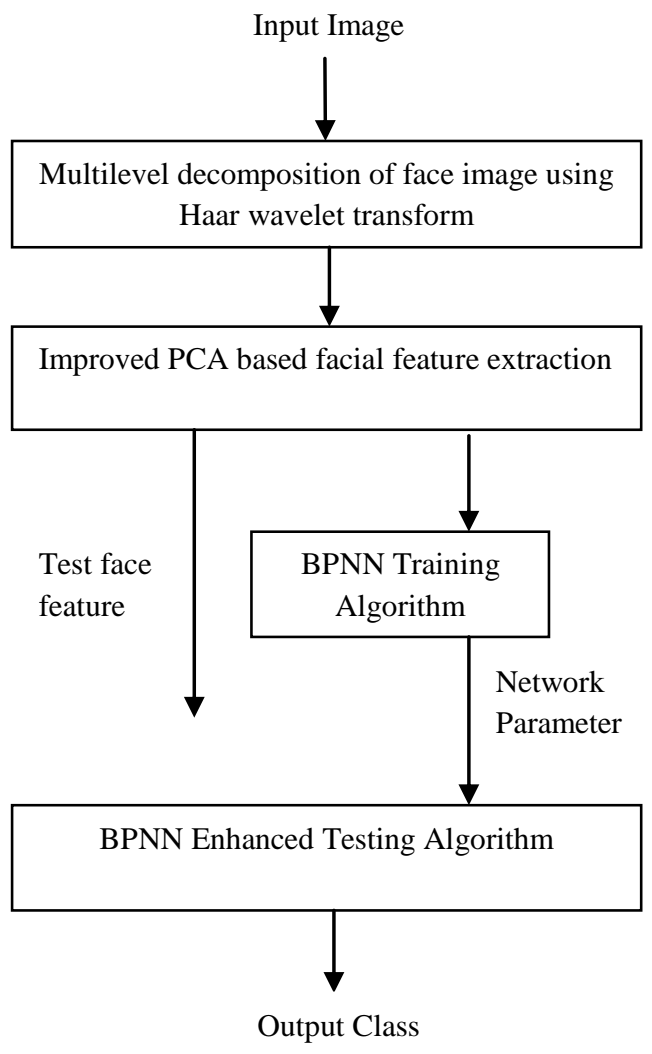

Fig 7: Proposed Efficient Face Recognition System 


\section{SIMULATED RESULTS AND THEIR DISCUSSIONS}

For this implementation MATLAB version 7.8.0.347 is followed to perform the simulation of the proposed algorithms. The size of the original image is considered to be $\mathrm{m} * \mathrm{n}$. The compiled MATLAB code presents the following consequences for the proposed approach. Firstly an image is inputted and Haar wavelet transform decomposes the image into several subband images.

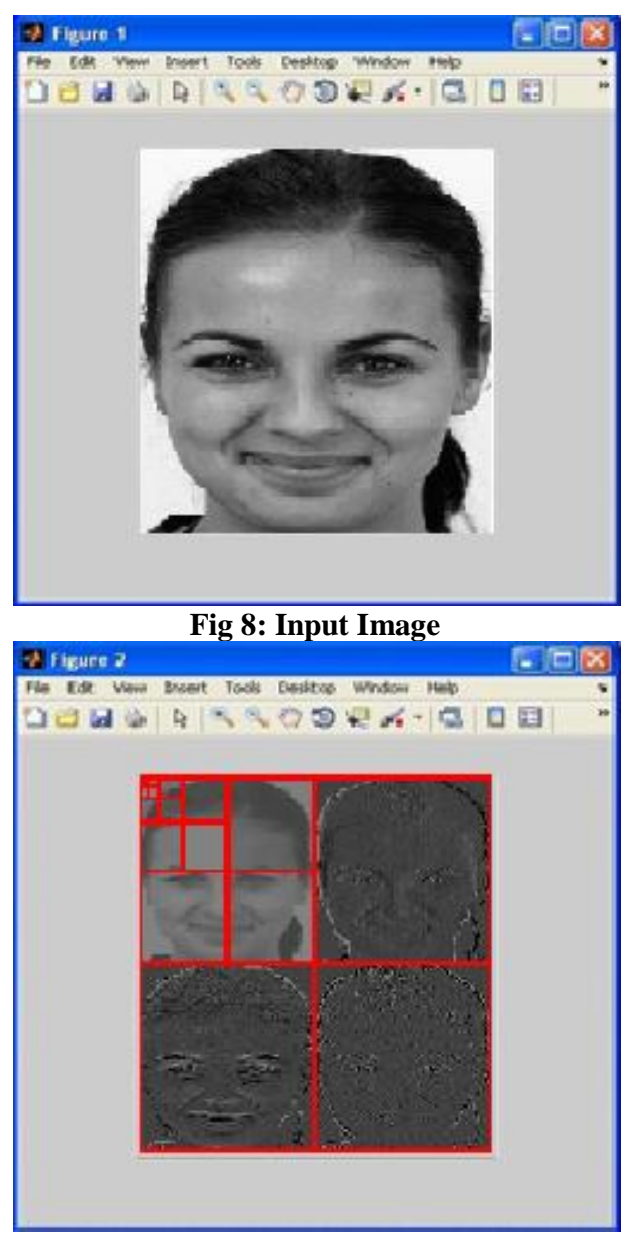

Fig 9: Multi level decomposed Image using Haar Wavelet Transform

Also, the histograms of input image and decomposed image are displayed here that defines the coefficients analyzed.

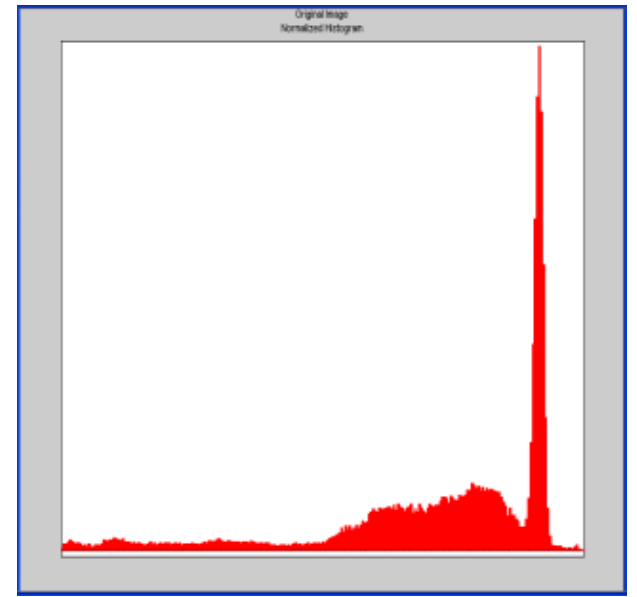

Fig 10: Original Image histogram

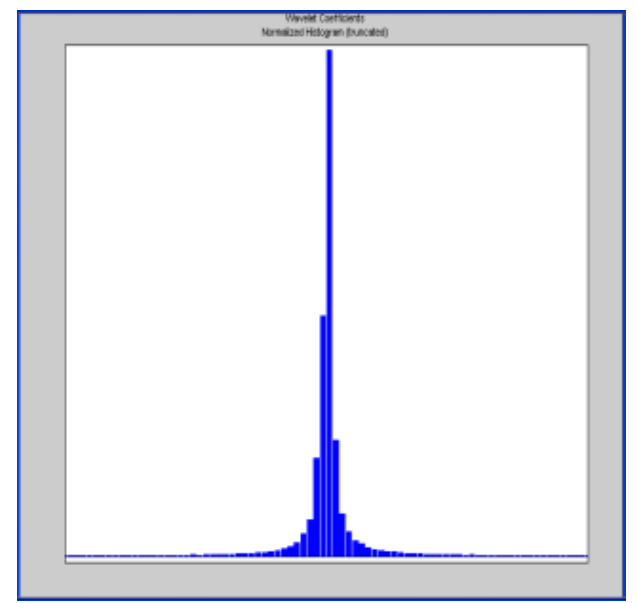

Fig 11: Decomposed image histogram

All the images in the database are numbered in the database so select a number for matching it up with the database.

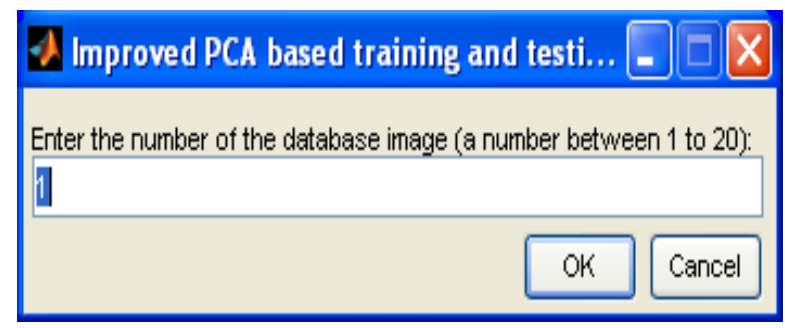

Fig 12: Input the number of database face image 


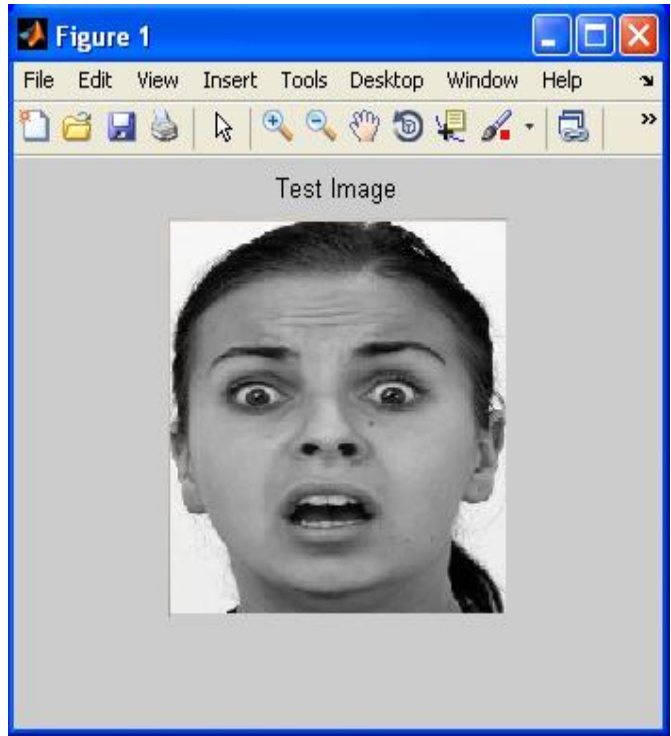

Fig 13: The image of train database being compared

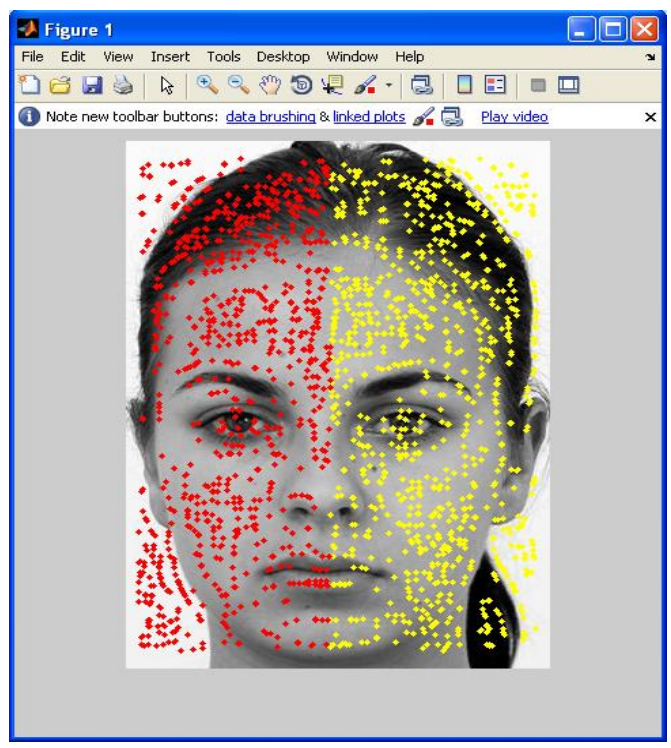

Fig 14: Features are extracted from face image

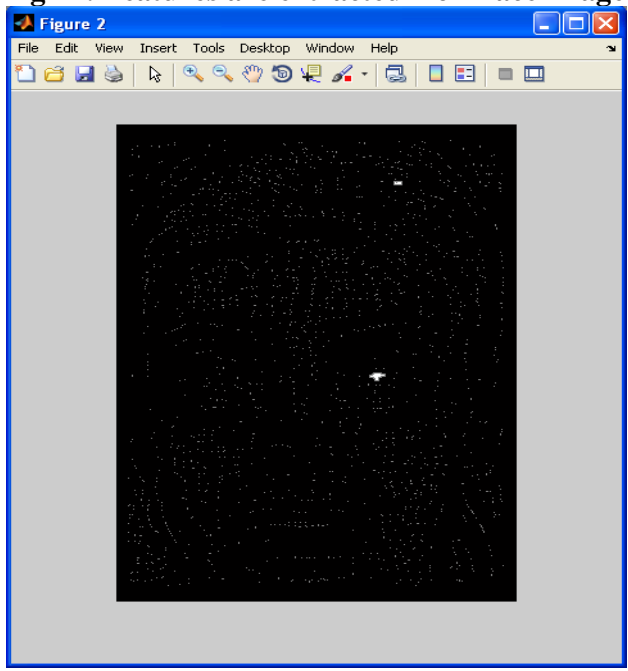

Fig 15: Corresponding classified features

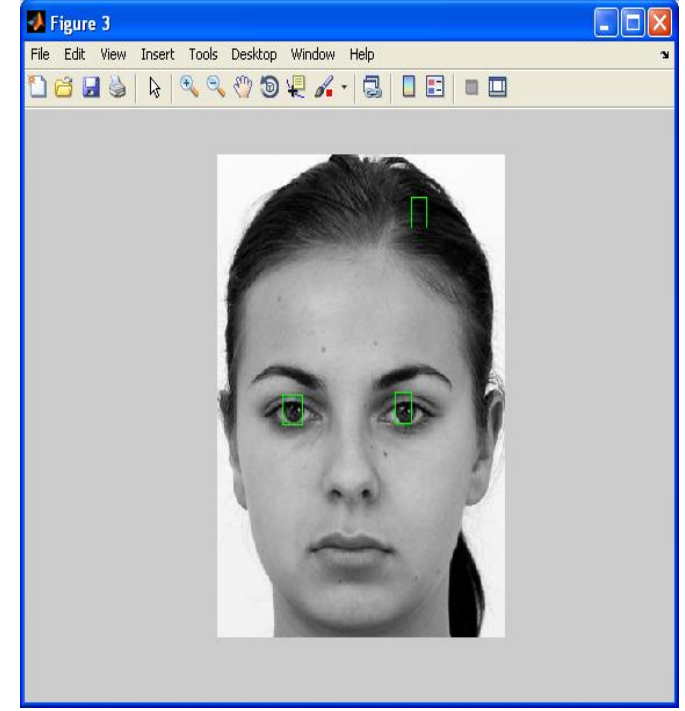

Fig 16: Recognized image

Recognition rate is the rate of measurement of correctness of the image being recognized. Using this combined approach recognition rate is increased to a much greater extend compared to other previously existing techniques. Hence, the efficiency of the system is improved.

Recognition rate $=($ The number of recognized images $) /$ (The number of testing images)

Elapsed time is the time of processing the feature extraction process and recognition of the image. Here elapsed time varies between 70 seconds to 200 seconds.

Elapsed time $=($ Run time for all testing images $) /($ The number of testing images)

\section{CONCLUSION}

In this paper, a hybrid approach is proposed for the efficient and secure designing of a robust face recognition system. The proposed approach reduces the computation load to distinguish an image from the database. Firstly multilevel decomposition using Haar wavelet transform has been implemented on an image to break up it into multilevel sub image bands. After that improved PCA is applied on the subband images for extracting Eigen values. Then highest eigen vector is calculated from these eigen values which is being used for classification and recognition of images using BPNN approach which is rich enough to inhibit the consequences of varying pose and illumination conditions.

Hence the proposed hybrid approach formulates a much reliable and accurate approach in comparison of the other existing techniques of recognition [14]. It also cuts down the time required for the execution of image classification and its recognition from the images of test database. Hence this approach enhances the acceptance ratio during the searching of images from the database and thus makes the system more secure and reliable from unauthorized as compared to our previous approach. 


\section{FUTURE DIRECTIONS}

In the future, an outlook will be to make the system learn with other prominent databases that poses great variations in the lighting conditions and the positioning of the head. Also, other factors can be included for the optimization of pre-processing methods on am image. Along with this, acknowledgement of people in real-time is needed to be done and in much less restrained situations to make the system more secure and reliable from the unauthorized accesses.

\section{ACKNOWLEDGMENT}

The authors would like to thank CS \& IT Department of Moradabad Institute of Technology, Moradabad and Computer Science \& Engineering Department of IFTM University, Moradabad for supporting and guiding this research project.

\section{REFERENCES}

[1] (2001) The Wikipedia website. [Online]. Available: http://en.wikipedia.org/wiki/Facial_recognition_syste $\mathrm{m}$.

[2] P.Latha, Dr.L.Ganesan, Dr.S.Annadurai, "Face Recognition using Neural Networks," Signal Processing, International Journal (SPIJ) Volume (3), Issue (5), pp. 153-160.

[3] Mohammod Abul Kashem, Md. Nasim Akhter, Shamim Ahmed, Md. Mahbub Alam, "Face Recognition System Based on Principal Component Analysis (PCA) with Back Propagation Neural Networks (BPNN)," International Journal of Scientific \& Engineering Research Volume 2, Issue 6, June2011.

[4] S.Adebayo Daramola, O. Sandra Odeghe, "Facial Expression Recognition using Neural Network -An Overview," International Journal of Soft Computing and Engineering (IJSCE), Volume-2, Issue-1, March 2012, pp. 224-227.

[5] Mayank Agarwal, Himanshu Agrawal, Nikunj Jain, Mr. Manish Kumar, "Face Recognition using Principle Component Analysis, Eigenface and Neural Network," Signal Acquisition and Processing, IEEE International Conference on, 2010, pp. 310-314.

[6] Raman Bhati, Sarika Jain, Nilesh Maltare, Durgesh Kumar Mishra, "A Comparative Analysis of Different
Neural Networks for Face Recognition Using Principal Component Analysis, Wavelets and Efficient Variable Learning Rate," Computer \& Communication Technology(ICCCT), IEEE Int'l Conf on, 2010 , pp. $526-531$.

[7] Jong-Min Kim, Myung-A Kang, "A Study of Face Recognition using the PCA and Error BackPropagation," Intelligent Human-Machine Systems and Cybernetics, IEEE Second International Conference on, 2010, pp. 241-244.

[8] Amir Benzaoui, Houcine Bourouba, Abdelhani Boukrouche, "System for Automatic Faces Detection," Image Processing Theory, Tools and Applications, IEEE, 2012.

[9] Ki-Chung Chung, Seok Cheol Kee, Sang Ryong Kim, "Face Recognition using Principal Component Analysis of Gabor Filter Responses".

[10]M.Koteswara Rao, K.Veera Swamy, K.Anitha sheela, "Face recognition using DWT and eigenvectors," Emerging Technology Trends in Electronics, Communication and Networking, 1st International Conference on, IEEE, 2012.

[11]Swarup Kumar Dandpat, Prof. Sukadev Meher, "Performance Improvement for Face Recognition Using PCA and Two-Dimensional PCA," Computer Communication and Informatics (ICCCI -2013), International Conference on, Jan. $04-06,2013$, Coimbatore, INDIA, IEEE, 2013.

[12]Himanshu S. Bhatt, Samarth Bharadwaj, Richa Singh, Mayank Vatsa, "Recognizing Surgically Altered Face Images Using Multiobjective Evolutionary Algorithm," Information Forensics And Security, Vol. 8, No. 1, IEEE transactions on, Jan 2013, pp. 89-100.

[13]Pushpaja V. Saudagare, D.S. Chaudhari, "Efficient Face Recognition System using Artificial Neural Network," International Journal of Computer Applications, Volume 41- No.21, March 2012, pp. 1215 .

[14]Prachi Agarwal, Naveen Prakash, "An efficient Back Propagation Neural Network based Face Recognition System using Haar Wavelet Transform and PCA," International Journal of Computer Science and Mobile Computing (IJCSMC), vol. 2, issue. 5, May 2013, pp. $386-395$. 